\title{
Cambios histológicos en muestras de agrandamientos gingivales obtenidas a través de biopsias con electrobisturí y bisturí convencional
}

\author{
Histological changes in samples of gingival overgrowth biopsies \\ obtained through conventional scalpel and electro surgical scalpel
}

Herrera Herrera A*, Díaz Caballero A**, Barrios García L***, Fang Mercado LC****

\section{RESUMEN}

Antecedentes: Es poco lo que se dice en la literatura sobre cambios histológicos observados en la encía y aun menos comparándolo en caso de agrandamiento gingival obtenido de tejidos humanos. Al realizar estos recortes surge la pregunta si existe alguna diferencia histológica al comparar las muestras obtenidas con electrobisturí y bisturí convencional.

Métodos: Se implementó un estudio descriptivo comparativo, sobre 21 pacientes obteniendo 90 muestras. La asignación de los sitios quirúrgicos para cada uno de los tratamientos se hizo con la estrategia de control cruzado, evaluando ambos tratamientos en el mismo sujeto (electrobisturí y bisturí convencional), en forma aleatoria en ambas hemiarcadas. Los datos se incluyeron en una base de datos en Excel, se analizaron en un software estadístico STATA 9.1. Se utilizó el test exacto de Fischer, con significancia p=0,05.

Resultados: Se observó carbonización y coagulación del colágeno en el 72,7\% siendo superior en las muestras obtenidas con electrobisturí en el tejido conectivo, el 79,5\% de diferencia de carbonización siendo superior con el electrobisturí en el tejido epitelial. Con respecto al bisturí convencional en el tejido conectivo en un 95,6\% esta inflamación estaba mediada por linfocitos y células plasmáticas y solo en un 17,8\% se encontró presencia de neutrófilos y con respecto al electrobisturí en el tejido conectivo el $100 \%$ de las muestras presentaron inflamación, el cual estaba constituido por un 97,8\% de linfocitos y células plasmáticas, solo un 37,8\% de neutrófilos. Conclusiones: Se observaron diversos fenómenos histológicos en las biopsias de encía obtenidas tanto con bisturí convencional como con electrobisturí que ayudan a la comprensión de las ciencias básicas para futuras investigaciones y así poder llegar a la práctica clínica. Se observaron daños en las biopsias tanto en los cortes de bisturí convencional como con los cortes de bisturí eléctrico, que en oportunidades hicieron invalorables las muestra.

Palabras clave: Estudio comparativo, gingivectomía, biopsia, histología comparada (Decs Bireme).

* Odontóloga. Universidad de Cartagena. Joven investigadora grupo de investigaciones GITOUC. Facultad de Odontología Universidad de Cartagena.

** Odontólogo. Universidad de Cartagena. Especialista en Periodoncia Universidad Javeriana. Magister en Educación Universidad del Norte. Estudiante de doctorado en Ciencias Biomédicas Universidad de Cartagena. Docente titular, Universidad de Cartagena. Director Grupo de investigaciones GITOUC.

*** Médica. Universidad de Cartagena. Especialista en patología Universidad de Cartagena. Profesora asistente Universidad de Cartagena.

**** Odontólogo. Universidad de Cartagena. Joven investigador grupo de investigaciones GITOUC. Facultad de Odontología. Universidad de Cartagena. 


\section{SUMMARY}

Background. Literature Reports are scarce regarding histological changes observed on gingival tissues, but they are scarcer when gingival overgrowth from human tissues are considered. When histological slides are compared, the question arises if there are histological differences between samples obtained with electrosurgical versus conventional scalpel.

Methods. A comparative study was performed in 21 patients, 90 samples. Cross control strategy was used to allocate the surgical site for each treatment, facilitating to evaluate both treatments in the same subject (electrosurgical versus conventional scalpel) in a random manner for both arches. Data was entered in an Excel data base and analyzed with STATA 9.1 statistical software. Fischer's exact test, $\mathrm{p}=0.05$ significance level was used. Results. Collagen burning and coagulation were observed in $72.7 \%$ of the cases, being higher for connective tissue samples obtained with electrosurgical scalpel, 79.5\% regarding burning, being higher with electrosurgical scalpel for epithelial tissue. Connective tissue showed inflammation in $95.6 \%$ of the cases for conventional scalpel, mediated by lymphocytes and plasmatic cells; neutrophils were seen in $17.8 \%$ of the sample and inflammation was seen in $100 \%$ of the samples for electrosurgical and conventional scalpels, which evidenced 97.8\% lymphocytes and plasmatic cells and $37.8 \%$ neutrophils.

Conclusions. Different histological phenomena were observed in gingival biopsies obtained with electrosurgical versus conventional scalpel; this facilitates understanding gingival overgrowth associated with orthodontic treatment. Some damage was observed in several biopsy samples which in some cases rendered the samples unusable for histological evaluation..

Key words: Comparative study, gingivectomy, biopsy, comparative histology (MESH).

Fecha de recepción: 30 de marzo de 2011.

Aceptado para publicación: 30 de junio de 2011.

Herrera Herrera A, Díaz Caballero A, Barrios García L, Fang Mercado LC. Cambios histológicos en muestras de agrandamientos gingivales obtenidas a través de biopsias con electro bisturí y bisturí convencionalucido. $A v$. Odontoestomatol 2012; 28 (3): 141-150.

\section{INTRODUCCIÓN}

En Odontología el uso de exámenes auxiliares o complementarios son de gran importancia ya que juegan un papel determinante en el diagnóstico de patologías orales, éstos permiten corroborar la percepción clínica a partir de las concepciones y prácticas de las ciencias básicas $(1,2)$. Un ejemplo de esto corresponde a la puntual aplicación de las biopsias, porque permiten corroborar desde el punto de vista histopatológico el diagnóstico definitivo de una lesión (1). Sin embargo en ningún momento las biopsias sustituirán a la exploración minuciosa y exhaustiva que caracterizan al examen clínico $(3,4)$.

La conducta terapéutica del odontólogo debe basarse en diagnósticos certeros, logrados por el conocimiento clínico de las diversas afecciones y corrobo- rado por el estudio histopatológico de estas, además de la fundamentación científica, lecturas complementarias, la experiencia profesional y evidencias clínicas de estudios serios y continuados, que facilitan un pensamiento complejo y estructurado (5) y le permitirán tomar decisiones terapéuticas adecuadas, partiendo desde las concepciones de las ciencias básicas, hasta llegar a la aplicación de estas en la clínica odontológica $(6,7)$.

Al realizar gingivectomías en individuos con agrandamiento gingival se puede optar por usar bisturí convencional o electrobisturí, sin embargo es una incógnita que poco se conoce y se reporta en la literatura, cual de las dos opciones es ideal para tal procedimientos. Por tanto el objetivo del presente estudio fue caracterizar los cambios histológicos que se presentaron en el tejido gingival obtenido a través de biop- 
sias realizadas con electrobisturí y bisturí convencional en pacientes con tratamiento de ortodoncia.

\section{MATERIALES Y MÉTODOS}

Se realizó un estudio descriptivo comparativo, con dos grupos de muestras gingivales obtenidas a partir de biopsias con electrobisturí (art-e1 bonart, en modalidad de corte- coagulación a intensidad numero 4 y punta eléctrica recta) y con bisturí convencional (Bard Parker número 3 con hoja de bisturí número 15). El tamaño de la muestra se calculó basado en la tendencia histórica $(8,9)$. Recolectando un resultado de 90 muestras de tejido gingival obtenidas en 21 sujetos que presentaron agrandamientos gingivales diagnosticados en las clínicas de ortodoncia de la facultad de Odontología de la Universidad de Cartagena.

Las muestras fueron obtenidas de las dos hemiarcadas de cada participante y se asignaron de forma aleatoria a las técnicas de obtención del tejido (45 para electrobisturí y 45 para bisturí convencional), con el fin de evitar la influencia de la ubicación del sitio en los resultados.

Previo a la selección se tuvo en cuenta los siguientes criterios: tejido gingival agrandado por motivos ortodónticos de pacientes que acuden a la clínica de postgrado de ortodoncia de la Universidad de Cartagena. No se incluyeron aquellos tejidos que provenían de embarazadas, pacientes fumadores, diabéticos e individuos con marcapasos.

\section{Instrumentos}

Para la recolección de información primero se diseñó y validó un formato tipo examen clínico y examen histológico. El formato histológico incluyó información de las variables principales del estudio en el tejido conectivo y tejido epitelial. Las cuales fueron realizadas e interpretadas por un examinador entrenado y calibrado a través de dos expertos en Periodoncia y Patología oral, al lograr una concordancia inter-observador mayor a $80 \%$.

Para el inicio de la recolección de los datos de las muestras histológicas se procedió a dividir en 2 los cuadrantes en la cavidad oral, utilizando uno de los cuadrantes recortes con electrobisturí y en el otro cuadrante recortes con bisturí convencional, tomando una muestra del sitio quirúrgico indicado por el experto en Periodoncia. Posteriormente el espécimen biológico se transfirió a un recipiente estéril conteniendo formol tamponado al $10 \%$ para luego llevar a laboratorio y proseguir con procedimientos de lavado, deshidratación, aclaramiento, montaje en parafina y recortes con el micrótomo que se realizó con un grosor de 5 micrómetros, se siguió con la pesca de las muestras en los porta objetos y terminar con la coloración con base de hematoxilina- eosina para poder observar las características detenidamente con el microscopio óptico binocular en un rango de $10 \mathrm{x}$ a 40x (10). Es importante resaltar que para la interpretación de resultados, las muestras fueron codificadas, evitando cualquier conocimiento del evaluador en histopatología, en cuanto al tipo de técnica que fue usada para su obtención, estando enmascarado todo el tiempo y de esta forma evitar sesgos en la obtención de los hallazgos.

\section{Análisis estadístico}

Después de la recolección de datos se procedió a incluirlos en una base de datos en Excel versión para Windows 7, luego se analizaron en un software estadístico STATA 9.1 (StataCorp, 4905 Lakeway Drive, College Station, Texas 77845 (USA). Previo al análisis se comprobó la normalidad de los datos a través de la prueba de Shapiro-Will, encontrándose una distribución diferente a la normal, lo que ameritó el uso de pruebas no paramétricas. Después de realizar esto se interpretaron los datos utilizando pruebas estadísticas descriptivas como la media y desviación estándar, la distribución de frecuencia con valores absolutos y relativos, asumiendo intervalos de confianza del 95\%. Para la comparación de las dos técnicas con respecto a cada una de las variables se utilizó el test exacto de Fischer, teniendo en cuenta que se trabajó con una significancia $p=0,05$.

\section{RESULTADOS}

En el estudio se escogió una muestra de 21 pacientes con un promedio de edad de 18,6 años $(\mathrm{DE}=3,9)$, 
de los cuales se obtuvieron 90 cortes gingivales. Con respecto al bisturí convencional en el tejido conectivo: el 97,8\% de las muestras no presentaron coagulación del colágeno y carbonización, pero es importante decir que el 97,8\% si presentó inflamación del conectivo, en un 95,6\% esta inflamación estaba mediada por linfocitos, células plasmáticas y solo en un $17,8 \%$ se observó presencia de neutrófilos. Por otro lado con esta misma técnica en el tejido epitelial: el 95,4\% de las muestras no presentaron carbonización y el $82,2 \%$ no presentaron coagulación del colágeno en el epitelio, pero en un $86,7 \%$ presentaron paraqueratosis y en un $82,2 \%$ acantosis, el $62,2 \%$ presentó espongiosis y en un $86,7 \%$ de las muestras no se encontraron presencia de balonización e hiperqueratosis (tablas $1 \mathrm{y} 2$ ).

De acuerdo a la condición de invalorable con bisturí convencional en el tejido conectivo, en nueve muestras no se pudieron observar sus características histológicas. De estas, en el 11,1\% no se apreciaron las características que evaluaban la coagulación del colágeno, carbonización, inflamación, presencia de linfocitos, células plasmáticas, neutrófilos e inflamación mixta, mientras que para infiltrado inflamatorio fue del 22,2\%. En el tejido epitelial, el 12,7\% fueron invalorables para la característica de espongiosis; 7,3\% para paraqueratosis y acantosis; $10,9 \%$ para balonización, atrofia, hiperqueratosis, redes de cresta, hiperplasia y exocitosis de leucocitos y solo el $3,6 \%$ para carbonización y coagulación del colágeno (tablas 3 y 4).

Con respecto al electrobisturí en el tejido conectivo el $71,1 \%$ presentó carbonización y coagulación del colágeno, el $100 \%$ de las muestras presentaron inflamación, el cual estaba constituido por un 97,8\% de linfocitos y células plasmáticas y solo un $37,8 \%$ de neutrófilos. Con esta técnica en el tejido epitelial: se presentó $77,8 \%$ de carbonización y coagulación del colágeno, en un $80 \%$ se evidenció paraqueratosis y espongiosis con exocitosis de leucocitos en solo $35,6 \%$. Es importante recalcar que en $71,1 \%$ fue para la ausencia de hiperqueratosis, y en un $57,8 \%$ hubo ausencia de acantosis e hiperplasia (tablas 1 y 2 ).

De acuerdo a las muestras invalorables en el tejido conectivo, en ocho muestras no se observaron sus características histológicas. De las cuales, el 12,5\% no se evidenciaron para coagulación del colágeno, carbonización, presencia de linfocitos, células plasmáticas, neutrófilos e inflamación mixta; el 25\% para infiltrado inflamatorio. En este mismo sentido, los hallazgos para inflamación se observaron en todas las muestras. En el tejido epitelial, el 13,9\% de las muestras fueron invalorables para la característica redes de cresta; $11,5 \%$ para exocitosis de leucocitos; $10,7 \%$ para espongiosis, hiperplasia, atrofia e hiperqueratosis; $13,1 \%$ para balonización; 9,8\% para acantosis y $7,4 \%$ fueron invalorable para la característica paraqueratosis (tablas 3 y 4 ).

\section{ANÁLISIS BIVARIADO}

\section{Tejido conectivo}

Se encontró una diferencia del 72,7\% (IC 95\%; 59,5$85,8 ; p=0,000)$, con respecto a la coagulación del colágeno y carbonización, siendo superior para las muestras que fueron obtenidas con el electrobisturí en comparación con el bisturí convencional, donde no se presenciaron casos. Adicional a esto, con relación a la inflamación y los linfocitos no hubo diferencias.

Al tener en cuenta los neutrófilos, las diferencias fueron del 20,4\%, a favor del electrobisturí (IC 95\%; 0,21$38,8)$, también es de notar que la inflamación mixta presentó una diferencia de $22,7 \%$ a favor del electrobisturí (IC 95\%; 0,47-4,07) y en el infiltrado inflamatorio se presentó en $18,6 \%$ a favor del electrobisturí (IC 95\%; -0,18-39,0) (tabla 5).

\section{Tejido epitelial}

Entre los resultados más relevantes siendo superior para el electrobisturí se encontró carbonización con una diferencia del 79,5\% (IC 95\%; 67,6-91,4), al igual para la coagulación del colágeno se presento diferencia del $65,6 \%$ (IC 95\%; 49,8-81,3) y para atrofia diferencia del 11,6\% (IC 95\%; -5,5-28,8), por el contrario siendo superior para el bisturí convencional se observó hiperplasia con una diferencia sobre el electrobisturí de 22,2\% (IC 95\%; -42,7- -1,7), también se observó espongiosis con diferencia de $23,6 \%$ (IC 95\%; $45,9-1,4)$ y con una diferencia de $15,6 \%$ se presenció redes de cresta (IC 95\%;-39,1- -7,8) (tabla 6). 


\section{TABLA 1. DESCRIPCIÓN HISTOLÓGICA DE LOS CAMBIOS PRESENTES EN EL TEJIDO CONECTIVO CON ELECTROBISTURÍ Y BISTURÍ CONVENCIONAL}

\begin{tabular}{|c|c|c|c|c|c|}
\hline \multicolumn{3}{|c|}{ ELECTROBISTURI } & \multicolumn{3}{|c|}{ BISTURI CONMENGONAL } \\
\hline & \multirow[b]{2}{*}{ RERUENAA $n=45$} & \multirow{3}{*}{ PORCENTAJE (\%) } & \multicolumn{3}{|c|}{ FRECUENCIA } \\
\hline & & & & $n=45$ & PORCENTAJE (\%) \\
\hline \multicolumn{5}{|c|}{ COAGULACION DEL COLAGENO } & \\
\hline PRESENTE & 32 & 71,1 & PRESENTE & 0 & 0 \\
\hline AUSENTE & 12 & 26,7 & AUSENTE & 44 & 97,8 \\
\hline INVALORABLE & 1 & 2,2 & INVALORABLE & 1 & 2,2 \\
\hline \multicolumn{6}{|c|}{ CARBONIZACIÓN } \\
\hline PRESENTE & 32 & 71,1 & PRESENTE & 0 & 0 \\
\hline AUSENTE & 12 & 26,7 & AUSENTE & 44 & 97,8 \\
\hline INVALORABLE & 1 & 2,2 & INVALORABLE & 1 & 2,2 \\
\hline \multicolumn{6}{|l|}{ INFLAMACION } \\
\hline PRESENTE & 45 & 100 & PRESENTE & 44 & 97,8 \\
\hline AUSENTE & 0 & 0 & AUSENTE & 0 & 0 \\
\hline INVALORABLE & 0 & 0 & INVALORABLE & 1 & 2,2 \\
\hline \multicolumn{6}{|l|}{ unFOATOS } \\
\hline PRESENTE & 44 & 97,8 & PRESENTE & 44 & 97,8 \\
\hline AUSENTE & 0 & 0 & AUSENIE & 0 & 0 \\
\hline INVALORABLE & 1 & 2,2 & INVALORABLE & 1 & 2,2 \\
\hline \multicolumn{6}{|c|}{ CELULASPLASMATICAS } \\
\hline PRESENTE & 44 & 97,8 & PRESENTE & 44 & 97,8 \\
\hline AUSENTE & 0 & 0 & AUSENIE & 0 & 0 \\
\hline INVALORABLE & 1 & 2,2 & INVALORABLE & 1 & 2,2 \\
\hline \multicolumn{6}{|l|}{ NEUTROHLOS } \\
\hline PRESENTE & 17 & 37,8 & PRESENTE & 8 & 17,8 \\
\hline AUSENTE & 27 & 60 & AUSENIE & 36 & 80 \\
\hline INVALORABLE & 1 & 2,2 & INVALORABLE & 1 & 2,2 \\
\hline \multicolumn{6}{|l|}{ MIXTO } \\
\hline PRESENTE & 17 & 37,8 & PRESENTE & 7 & 15,6 \\
\hline AUSENTE & 27 & 60 & AUSENIE & 37 & 82,2 \\
\hline INVALORABLE & 1 & 2,2 & INVALORABLE & 1 & 2,2 \\
\hline \multicolumn{6}{|c|}{ INFILTRADOINFLAMATORIO } \\
\hline PRESENIE & 29 & 64,4 & PRESENTE & 21 & 46,7 \\
\hline AUSENTE & 14 & 31,1 & AUSENTE & 22 & 48,9 \\
\hline INVALORABLE & 2 & 4,4 & INVALORABLE & 2 & 4,4 \\
\hline
\end{tabular}




\section{TABLA 2. DESCRIPCIÓN HISTOLÓGICA DE LOS CAMBIOS PRESENTES EN EL TEJIDO EPITELIAL CON ELECTROBISTURÍ Y BISTURÍ CONVENCIONAL}

TEJIDO EPITELIAL

\begin{tabular}{|c|c|c|c|c|c|c|}
\hline \multicolumn{4}{|c|}{$\begin{array}{l}\text { ELECTROBISTURI } \\
\end{array}$} & \multicolumn{3}{|c|}{ 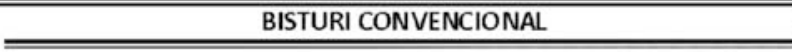 } \\
\hline & RRECUENCIA & $n=45$ & PORCENTAIE $(\%)$ & & FRECUENCIA $n=45$ & PORCENTAJE (\%) \\
\hline \multicolumn{7}{|c|}{ COAGULACION DEL COLAGENO } \\
\hline PRESENTE & & 35 & 77,8 & PRESENTE & 6 & 13,3 \\
\hline AUSENTE & & 9 & 20 & AUSENTE & 37 & 82,2 \\
\hline INVALORABLE & & 1 & 2,2 & INVALORABLE & 2 & 4,4 \\
\hline \multicolumn{7}{|c|}{ CARBONIZACIÓN } \\
\hline PRESENTE & & 35 & 77,8 & PRESENTE & 0 & 0 \\
\hline AUSENTE & & 9 & 20 & AUSENTE & 43 & 95,6 \\
\hline INVALORABLE & & 1 & 2,2 & INVALORABLE & 2 & 4,4 \\
\hline \multicolumn{7}{|c|}{ PARAQUERATOSIS } \\
\hline PRESENTE & & 36 & 80 & PRESENTE & 39 & 86,7 \\
\hline AUSENTE & & 0 & 0 & AUSENTE & 2 & 4,4 \\
\hline INVALORABLE & & 9 & 20 & INVALORABLE & 4 & 8,9 \\
\hline \multicolumn{7}{|l|}{ ACANTOSIS } \\
\hline PRESENTE & & 32 & 71,1 & PRESENTE & 37 & 82,2 \\
\hline AUSENTE & & 1 & 2,2 & AUSENTE & 4 & 8,9 \\
\hline INVALORABLE & & 12 & 26,7 & INVALORABLE & 4 & 8,9 \\
\hline \multicolumn{7}{|l|}{ BALONIZACION } \\
\hline PRESENTE & & 2 & 4,4 & PRESENTE & 0 & 0 \\
\hline AUSENTE & & 27 & 60 & AUSENTE & 39 & 86,7 \\
\hline INVALORABLE & & 16 & 35,6 & INVALORABLE & 6 & 13,3 \\
\hline \multicolumn{7}{|l|}{ HIPERPLASIA } \\
\hline PRESENTE & & 6 & 13,3 & PRESENTE & 16 & 35,6 \\
\hline AUSENTE & & 26 & 57,8 & AUSENTE & 23 & 51,1 \\
\hline INVALORABLE & & 13 & 28,9 & INVALORABLE & 6 & 13,3 \\
\hline \multicolumn{7}{|l|}{ ATROFIA } \\
\hline PRESENTE & & 7 & 15,6 & PRESENTE & 4 & 8,9 \\
\hline AUSENTE & & 25 & 55,6 & AUSENTE & 35 & 77,8 \\
\hline INVALORABLE & & 13 & 28,9 & INVALORABLE & 6 & 13,3 \\
\hline \multicolumn{7}{|c|}{ HIPERQUERATORIS } \\
\hline PRESENTE & & 0 & 0 & PRESENTE & 0 & 0 \\
\hline AUSENTE & & 32 & 71,1 & AUSENTE & 39 & 86,7 \\
\hline INVALORABLE & & 13 & 28,9 & INVALORABLE & 6 & 13,3 \\
\hline \multicolumn{7}{|l|}{ ESPONJIOSIS } \\
\hline PRESENTE & & 16 & 35,6 & PRESENTE & 28 & 62,2 \\
\hline AUSENTE & & 16 & 35,6 & AUSENTE & 10 & 22,2 \\
\hline INVALORABLE & & 13 & 28,9 & INVALORABLE & $\frac{7}{7}$ & 15,6 \\
\hline \multicolumn{7}{|c|}{ EXOCITOSIS DE LEUCOSITOS } \\
\hline PRESENTE & & 16 & 35,6 & PRESENTE & 14 & 31,1 \\
\hline AUSENTE & & 15 & 33,3 & AUSENTE & $\begin{array}{l}14 \\
25\end{array}$ & 55,6 \\
\hline INVALORABLE & & 14 & 31,1 & INVALORABLE & $\begin{array}{r}25 \\
6\end{array}$ & 13,3 \\
\hline \multicolumn{7}{|c|}{ REDES DE CRESTA } \\
\hline PRESENTE & & 24 & 53,3 & PRESENTE & 34 & 75,6 \\
\hline AUSENTE & & 4 & 8,9 & AUSENTE & 5 & 11,1 \\
\hline INVALORABLE & & 17 & 37,8 & INVALORABLE & 6 & 13,3 \\
\hline
\end{tabular}




\begin{tabular}{|c|c|c|}
\hline \multicolumn{3}{|c|}{$\begin{array}{c}\text { TABLA 3. DESCRIPCIÓN DE LOS } \\
\text { PORCENTAJES INVALORABLES PARA CADA } \\
\text { VARIABLE POR DAÑOS EN LA OBTENCIÓN } \\
\text { DE LA BIOPSIA PARA LA IMAGEN EN EL } \\
\text { MICROSCOPIO ELECTRÓNICO EN EL } \\
\text { TEJIDO CONECTIVO }\end{array}$} \\
\hline \multicolumn{3}{|c|}{ Muestras invalorables en el tejido conectivo } \\
\hline & Electrobisturí & Convencional \\
\hline $\begin{array}{l}\text { Coagulación del } \\
\text { colágeno } \\
\text { Carbonización } \\
\text { Inflamación } \\
\text { Linfocitos } \\
\text { Células plasmáticas } \\
\text { Neutrófilos } \\
\text { Mixto } \\
\text { Infil. Inflamatorio }\end{array}$ & $\begin{array}{c}12,5 \\
12,5 \\
0 \\
12,5 \\
12,5 \\
12,5 \\
12,5 \\
25\end{array}$ & $\begin{array}{l}11,1 \\
11,1 \\
11,1 \\
11,1 \\
11,1 \\
11,1 \\
11,1 \\
22,2\end{array}$ \\
\hline
\end{tabular}

\section{DISCUSIÓN}

En el siguiente estudio presentaron limitaciones al no evaluar respuesta inflamatoria y cicatrización de los pacientes por presentar dificultad en el seguimiento, pero se obtuvieron alcances importantes ya que al contrastar los estudios realizados por Jin JY, Lee SH, Yoon HJ, en el 2010 (11), en un estudio comparativo de diferentes herramientas de corte en

\begin{tabular}{|c|c|c|}
\hline \multicolumn{3}{|c|}{$\begin{array}{c}\text { TABLA 4. DESCRIPCIÓN DE LOS } \\
\text { PORCENTAJES INVALORABLES PARA CADA } \\
\text { VARIABLE POR DAÑOS EN LA OBTENCIÓN } \\
\text { DE LA BIOPSIA PARA LA IMAGEN EN EL } \\
\text { MICROSCOPIO ELECTRÓNICO EN EL } \\
\text { TEJIDO EPITELIAL }\end{array}$} \\
\hline \multicolumn{3}{|c|}{ Muestras invalorables en el tejido epitelial } \\
\hline & Electrobisturí & Convencion: \\
\hline $\begin{array}{l}\text { Coagulación del } \\
\text { colágeno } \\
\text { Carbonización } \\
\text { Paraqueratosis } \\
\text { Acantosis } \\
\text { Balonización } \\
\text { Hiperplasia } \\
\text { Espongiosis } \\
\text { Exocitosis de } \\
\text { leucocitos } \\
\text { Red de cresta } \\
\text { Atrofia } \\
\text { Hiperqueratosis }\end{array}$ & $\begin{array}{r}0,83 \\
0,83 \\
7,37 \\
9,83 \\
13,1 \\
10,6 \\
10,6\end{array}$ & $\begin{array}{r}3,6 \\
3,6 \\
7,2 \\
7,2 \\
19,9 \\
10,9 \\
12,7\end{array}$ \\
\hline
\end{tabular}

la mucosa oral, encontraron de manera clara que el bisturí convencional es el que mayores daños produce al usarse como medio de toma de muestra en la cavidad oral, que se producen daños histológicos que en oportunidades hacen inviables los especíme-

TABLA 5. ANÁLISIS BIVARIADO COMPARANDO CAMBIOS HISTOLÓGICOS PRESENTES CON ELECTROBISTURÍ VS CAMBIOS PRESENTES CON BISTURÍ CONVENCIONAL CON INTERVALOS DE CONFIANZA EN EL TEJIDO CONECTIVO

\begin{tabular}{|l|c|c|c|c|}
\hline \multicolumn{5}{|c|}{ Tejido conectivo } \\
\hline & Electrobisturí & Convencional & Diferencia & IC 95\% \\
\hline Coagulación del colágeno & 72,7 & 0 & 72,7 & $59,5-85,8$ \\
Carbonización & 72,7 & 0 & 72,7 & $59,5-85,8$ \\
Inflamación & 1 & 1 & 0 & 0 \\
Linfocitos & 1 & 1 & 0 & 0 \\
Células plasmáticas & 1 & 1 & 0 & 0 \\
Neutrófilos & 38,6 & 18,1 & 20,4 & $0,21-38,8$ \\
Mixto & 38,6 & 15,9 & 18,6 & $0,47-4,07$ \\
Infil. inflamatorio & 67,4 & 48,8 & $-0,18-39,0$ \\
\hline
\end{tabular}




\section{TABLA 6. ANÁLISIS BIVARIADO COMPARANDO CAMBIOS HISTOLÓGICOS PRESENTES CON ELECTROBISTURÍ VS CAMBIOS PRESENTES CON BISTURÍ CONVENCIONAL CON INTERVALOS DE CONFIANZA EN EL TEJIDO EPITELIAL}

\begin{tabular}{|l|c|c|c|c|}
\hline \multicolumn{5}{|c|}{ Tejido epitelial } \\
\hline & Electrobisturí & Convencional & Diferencia & IC 95\% \\
\hline Coagulación del colágeno & 79,5 & 13,9 & 65,5 & $49,8-81,3$ \\
Carbonización & 79,5 & 0 & 79,54 & $67,6-91,4$ \\
Paraqueratosis & 100 & 95,1 & 4,8 & $-1,7-11,4$ \\
Acantosis & 96,9 & 90,2 & 6,7 & $-4-17,5$ \\
Balonización & 6,8 & 0 & 6,8 & $-2,3-16,1$ \\
Hiperplasia & 18,7 & 41 & $-22,2$ & $-42,7--1,7$ \\
Espongiosis & 50 & 73,6 & $-23,6$ & $45,9--1,4$ \\
Exocitosis de leucocitos & 51,6 & 35,8 & 15,7 & $-7,4-38,8$ \\
Red de cresta & 53,5 & 69,2 & $-15,6$ & $-39,1-7,8$ \\
Atrofia & 21,8 & 10,2 & 11,6 & $-5,5-28,8$ \\
Hiperqueratosis & 0 & 0 & 0 & 0 \\
\hline
\end{tabular}

$\mathrm{p}=0,000$

nes obtenidos para el respectivo análisis histopatológico. Los mismos autores señalan que se necesitan muchos más estudios de este tipo, para establecer de forma clara la mayor efectividad de los diferentes tipos de bisturí en cavidad oral. Al contrastar las técnicas de obtención de las muestras, se establecen diferencias en cuanto a la tecnología utilizada, ya que estos autores utilizaron cortes con láser de diodo y su aplicabilidad en los análisis histopatológico, mientras que en el presente estudio se utilizó el bisturí convencional y el electrobisturí, los cuales son de uso más frecuente en este tipo de poblaciones.

De acuerdo a los resultados obtenidos por el grupo de investigación actual es factible determinar de forma racional que tanto el uso del bisturí convencional como el electrobisturí, pueden ser empleados de manera científica como método de realización de biopsias en gingivectomías de pacientes con agrandamientos gingivales, para observar cambios histológicos tanto en el epitelio como en el tejido conectivo.

En varios estudios, tanto en animales como en humanos, se comparan los efectos del bisturí convencional contra los del electrocauterio en diferentes tejidos, sin encontrar verdaderas diferencias ni clíni- cas ni estadísticas (11-16). En el actual estudio se pudo determinar que aunque se presentaron en el $71,1 \%$ carbonización y coagulación en el tejido conectivo y un $77,8 \%$ de carbonización en el epitelio, solo se observa en el borde y se pueden ver todas las características necesarias como linfocitos, células plasmáticas, neutrófilos, para determinar la presencia de inflamación y entre otras características como espongiosis, balonización, atrofia etc, las cuales se presenten en el epitelio. En este sentido, estos hallazgos son de difícil discusión, debido a que en la literatura no hay evidencia con peso científico arroje resultados estadísticos sobre presencia de estructuras celulares e inflamación, ni mucho permiten una cuantificación de estos.

Moskow BS, Polson AM en 1991 (17), realizaron un estudio donde establecen la cantidad de infiltrado inflamatorio observado en diversos especímenes de periodontitis, gingivitis y en encías sanas en humanos, pero no alcanzan a expresar un porcentaje en cada situación estudiada. Se establece entonces que la presencia de infiltrado celular inflamatorio es mucho mayor que lo reportado en previos estudios en encías sanas. De forma similar, en el presente estudio se pudo detectar que a pesar de que los especímenes evaluados provienen de tejido gingival 
de pacientes sometidos a procesos higiénicos periódicos durante su tratamiento de ortodoncia, se encontró la presencia de infiltrado inflamatorio con predominio de células linfocitarias y en diferentes niveles de penetración en conectivo. Lo que establece de manera veraz que se debe considerar importante la presencia de células inflamatorias en cada evento quirúrgico que se realice en las cirugías periodontales, lo que puede afectar la capacidad de respuesta tisular ante el tratamiento instaurado, a pesar de tener una condición de "salud clínica periodontal".

A la luz de las limitantes actuales, por medio del presente estudio se pudo determinar que las biopsias gingivales se pueden obtener tanto con bisturí convencional como con bisturí eléctrico y las muestras obtenidas mantendrían su capacidad de herramienta diagnostica a nivel histopatológico, se establece que no se dañan las muestras como tal, manteniendo la validez de la toma de biopsias tanto con un bisturí como con otro, ya que se pudo establecer que el mayor riesgo de dañar las muestras se presentan con la manipulación ex vivo por parte del operador. Además dentro las características observadas en el estudio en pacientes con agrandamiento gingival se resaltan la presencia de infiltrado inflamatorio crónico observada en todas las muestras histológicas, acompañadas de acantosis y exocitosis de leucocitos en el tejido epitelial, así como de carbonización, coagulación del colágeno e infiltrado inflamatorio en el tejido conectivo.

\section{BIBLIOGRAFÍA}

1. Fatima G, Sandesh N, Ravindra S, Kulkarni S. Moving beyond clinical appearance: the need for accurate histological diagnosis. Gen Dent. 2009 Sep-Oct;57(5):472-7; quiz 8-9, 535-6.

2. Montagna G, Raimondi S, Moro G, Uggetti C, Relini A, Magrini U, et al. Clinical, radiological, and biochemical features of a bilateral buttock amyloidoma emerging after 27 years of hemodialysis. Amyloid. 2009;16(3):115-21.

3. Hammerschmidt S, Wirtz H. Lung cancer: current diagnosis and treatment. Dtsch Arztebl Int. 2009 Dec;106(49):809-18; quiz 19-20.
4. Bruno JF, Bowers GM. Histology of a human biopsy section following the placement of a subepithelial connective tissue graft. Int $\mathrm{J}$ Periodontics Restorative Dent. 2000 Jun;20(3): 225-31.

5. Brown BR, Inglehart MR. Orthodontists' and orthodontic residents' education in treating underserved patients: effects on professional attitudes and behavior. J Dent Educ. 2009 May; 73(5):550-62.

6. Acero EJ, Amador Avila T, Granobles L, Torres S. Actividad "In Vivo" del cloruro de berberina frente a una leishmaniasis cutánea causada por la especie Leishmania mexicana mexicana 856/Ins. Revista Colombiana de química Bogotá (Colomba). 1995;24(2).

7. Stablein MJ, Silverglade LB. Comparative analysis of biopsy specimens from gingiva and alveolar mucosa. J Periodontol. 1985 Nov;56(11): 671-6.

8. Marín RH. Estudio comparativo de las características histológicas de encías clínicamente normales e inflamadas en niños.

9. Berti SU. Efecto de Tres Diferentes Enjuagues Bucales sobre la Mucosa Gingival Humana. Estudio Histológico. Investigación Clínica. 2009; 20(1).

10. Hernández-Chavarría F, Vargas-Montero M, Rivera $P$, Carranza $A$. Un método rápido de reprocesamiento para microscopia electrónica de cortes histológicos en parafina. Rev costarric cienc méd. 2001;22(1-2):51-6.

11. Jin JY, Lee SH, Yoon HJ. A comparative study of wound healing following incision with a scalpel, diode laser or Er, Cr: YSGG laser in guinea pig oral mucosa: A histological and immunohistochemical analysis. Acta Odontologica Scandinavica (0):1-7.

12. Dixon AR, Watkin DF. Electrosurgical skin incision versus conventional scalpel: a prospective trial. Journal of the Royal College of Surgeons of Edinburgh. 1990;35(5):299. 
13. Johnson CD, Serpell JW. Wound infection after abdominal incision with scalpel or diathermy. Scalpel.130(68):18-95.

14. Soares FM, Tarver EJ, Bimstein E, Shaddox LM, Bhattacharyya I. Gingival overgrowth in a child with arthrogryposis treated with a Er,Cr:YSGG laser: a case report. Pediatr Dent. 2009 JanFeb;31(1):8-13.

15. Tamarit-Borras M, Delgado-Molina E, Berini-Aytes L, Gay-Escoda C. Removal of hyperplastic lesions of the oral cavity. A retrospective study of 128 cases. Med Oral Patol Oral Cir Bucal. 2005 MarApr;10(2):151-62.

16. Sinha UK, Gallagher LA. Effects of steel scalpel, ultrasonic scalpel, CO2 laser, and monopolar and bipolar electrosurgery on wound healing in guinea pig oral mucosa. The Laryngoscope. 2003; 113(2):228-36.

17. Moskow BS, Poison AM. Histologic studies on the extension of the inflammatory infiltrate in human periodontitis. Journal of Clinical Periodontology. 1991;18(7):534-42.

\section{CORRESPONDENCIA}

Alejandra Herrera Herrera

Facultad de Odontología, Universidad de Cartagena. Campus de la Salud Zaragocilla.

Cartagena, Bolivar, Colombia

Correos electrónicos: aleja2304@hotmail.com, adiazc1@unicartagena.edu.co. 